Article

\title{
Design and Synthesis of Some New 1,3,4-Thiadiazines with Coumarin Moieties and Their Antioxidative and Antifungal Activity
}

\author{
Milan Čačić ${ }^{1, *}$, Valentina Pavićc ${ }^{2}$, Maja Molnar ${ }^{1}$, Bojan Šarkanj ${ }^{1}$ and Elizabeta Has-Schön ${ }^{2}$ \\ 1 Department of Applied Chemistry and Ecology, Faculty of Food Technology, J. J. Strossmayer \\ University, Franje Kuhača 20, Osijek 31 000, Croatia; E-Mails: maja.molnar@ptfos.hr (M.M.); \\ bojan.sarkanj@ptfos.hr (B.S.) \\ 2 Department of Biology, J. J. Strossmayer University, Cara Hadrijana 8/A, Osijek 31 000, Croatia; \\ E-Mails: valentina.pavic@biologija.unios.hr (V.P.); hasschon@biologija.unios.hr (E.H.-S.) \\ * Author to whom correspondence should be addressed; E-Mail: mcacic@ptfos.hr \\ Tel.: +385-31-224-342; Fax: +385-31-207-117.
}

Received: 12 December 2013; in revised form: 13 January 2014 / Accepted: 14 January 2014 / Published: 17 January 2014

\begin{abstract}
A series of newly disubstituted (compounds 4a,b) and trisubstituted 1,3,4-thiadiazines $\mathbf{5 a}-\mathbf{l}$ with various substituents was prepared utilizing different thiosemicarbazides and 3- $\alpha$-bromoacetylcoumarins as starting compounds. The structures of the synthesized 1,3,4-thiadiazines are elucidated and confirmed utilizing the corresponding analytical and spectroscopic data. All of the new thiadiazine derivatives were tested for their antioxidant activity, employing different antioxidant assays (DPPH scavenging activity, iron chelating activity, power reducing activity). Compounds $\mathbf{5 b}, \mathbf{5 f}, \mathbf{5 j}$ and $\mathbf{4 b}$ were proven to be the best DPPH radical scavengers, while compounds $\mathbf{5 h}$ and $\mathbf{5 j}$ have shown the best iron chelating activity. Thiadiazine derivatives were also tested on their antifungal activity against four mycotoxicogenic fungi, Aspergillus flavus, A. ochraceus, Fusarium graminearum and $F$. verticillioides. The best antifungal against $A$. flavus was proven to be compound $\mathbf{5 e}$, while compounds $4 \mathbf{a}$ and $\mathbf{5 c}$ were the best antifungals on $A$. ochraceus, and compound $\mathbf{5 g}$ showed the best antifungal activity on $F$. verticillioides.
\end{abstract}

Keywords: coumarin; 1,3,4-thiadiazines; thiosemicarbazides; antifungal activity; antioxidative activity 


\section{Introduction}

Coumarin (2-oxo-2H-chromene) and its derivatives represent one of the most important classes of compounds possessing numerous biological activities [1-3]. Some of these compounds have proven to be active as antibacterial [4-6], antifungal [7], anti-inflammatory [8], anticoagulant [9], anti-HIV [10] and antitumor agents [11]. Coumarin derivatives are widely used as additives in food and cosmetics [12], pharmaceuticals and optical brighteners [13] and laser dyes [14]. Coumarins have also proven to be an excellent antioxidants as well as antifungal agents [15].

In recent years, interest in thiadiazines has increased due to the high biological activity and broadspectrum action of their derivatives [16]. Many thiadiazines have been discovered with possible applications in medical practice as sedatives, antianxiety agents, antiasthmatic agents, anticonvulsants, myorelaxants, coronary vasodilators, and spasmolytics. Synthesis of the 1,3,4-thiadiazine system employing a reaction of $\alpha$-bromoacetophenone with thiosemicarbazide was first reported by Bose [17]. This procedure is characterized by the formation of a few heterocyclic isomers. Which isomer is formed depends on the $\mathrm{H}^{+}$-ion concentration in the system, the polarity of the solvent, the reaction temperature and on the substituents. Depending on whether condensation occurs at the $\mathrm{N}_{1}, \mathrm{~N}_{2}$, or $\mathrm{N}_{4}$ of the thiosemicarbazide, three different sulfur-containing heterocyclic rings are expected after ring closure: the $6 H-1,3,4$-thiadiazines, the 2-substituted imino-2,3-dihydrothiazol-3-amines, and the 3-substituted 2-hydrazono-2,3-dihydrothiazoles.

In addition, 1,3,4-thiadiazines may exist in three different tautomeric forms. Spectroscopic investigations suggest that the $6 \mathrm{H}$-form is preferred. The $4 \mathrm{H}$-form represents a potentially anti-aromatic $8 \pi$-system which can be transformed by valence isomerization into a thiahomopyrazole and by subsequent extrusion of sulfur into a pyrazole [18]. In continuation of our research program on the synthesis of novel heterocyclic compounds exhibiting antioxidative and biological activity, it was thought to be interesting to synthesize compounds containing both coumarin and thiadiazine groups in their structure.

\section{Results and Discussion}

\subsection{Chemistry}

In continuation of our ongoing research program to synthesize potentially biologically active 1,3,4thiadiazine derivatives, in a present report we describe a series of $6 H-1,3,4$-thiadizines (compounds 4a, 4b) and 4H-1,3,4-thiadiazines (compounds 5a-l). The reaction sequence for the synthesis of 5-(2-oxo2H-chromen-3-yl)-6H-1,3,4-thiadizin-2-aminium bromide (4a) and 3-(2-amino-6H-1,3,4-thiadiazin-5yl)-4-hydroxy- $2 H$-chromen-2-one (4b), is outlined in Scheme 1. 3-( $\alpha$-Bromoacetyl)coumarins 1a,b were prepared according to the earlier literature methods [19,20]. Reaction of 1a with thiosemicarbazide $\mathbf{2}$ in ethanol at room temperature as the first step, followed by refluxing of the ethanolic solution of the obtained product in the presence of $48 \% \mathrm{HBr}$ gave compound $\mathbf{4 a}$. Compound 4b was prepared from $\mathbf{1 b}$ and thiosemicarbazide in ethanol in a presence of small amount of $\mathrm{HCl}$.

The structures of the products $\mathbf{4 a}$ and $\mathbf{4 b}$ were inferred from their analytical and spectral data. Thus, their IR spectra showed characteristic absorption bands at 3426-3183 (NH; OH), 1714-1696 (lactone $\mathrm{C}=\mathrm{O}), 1617-1600(-\mathrm{C}=\mathrm{N}), 756-760(\mathrm{CH}-$ arom. $)$ and $(\mathrm{C}-\mathrm{S}-\mathrm{C})$ at $675 \mathrm{~cm}^{-1}$. The ${ }^{1} \mathrm{H}-\mathrm{NMR}$ spectra of 
compounds $4 \mathbf{a}$ and $\mathbf{4 b}$ exhibited singlets at $3.91\left(\mathrm{~s}, 2 \mathrm{H}, \mathrm{CH}_{2}\right), 4.80\left(\mathrm{~s}, 2 \mathrm{H}, \mathrm{NH}_{2} /-\mathrm{N}^{+} \mathrm{H}_{3}\right)$ and $14.68 \mathrm{ppm}$ (s, 1H, -OH).

Scheme 1. The reaction sequence for the synthesis of 5-(2-oxo- $2 H$-chromen-3-yl)- $6 H$ 1,3,4-thiadizin-2-aminium bromide (4a) and 3-(2-amino-6H-1,3,4-thiadiazin-5-yl)-4hydroxy-2H-chromen-2-one (4b).<smiles>[R]c1c(C(=O)CBr)c(=O)oc2ccccc12</smiles>

$\mathrm{R}_{1}$ : a) $\left.-\mathrm{H} ; \mathrm{b}\right)-\mathrm{OH}$<smiles>NNC(N)=S</smiles>

2

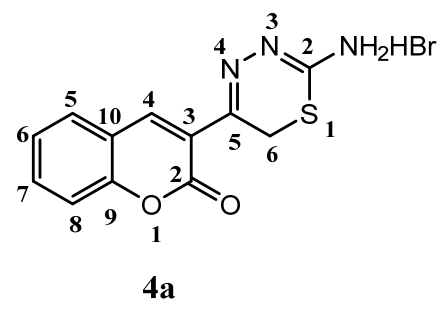

$\mathrm{C}_{2} \mathrm{H}_{5} \mathrm{OH} / \mathrm{H}^{+}$

2) $\mathrm{HBr} 48 \%$

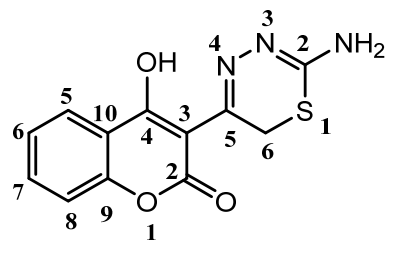

$4 b$

1,4-Thiosemicarbazide derivatives $\mathbf{3 a}-\mathbf{g}$ were synthesized as previously described [21] by reacting equimolar amounts of ethyl-, methyl- or phenylisothiocyanate with 4-hydroxybenzohydrazide, 2-(7-hydroxy-2-oxo-2H-chromen-4-yl)acetohydrazide and 2-(4-methyl-2-oxo-2H-chromen-7-yloxy)acetohydrazide.

The compounds 5a-l were prepared by heating 3- $\alpha$-bromoacetylcoumarins 1a,b with the corresponding 1,4-disubstituted thiosemicarbazides 3a-g in ethanol, with few drops of hydrochloric acid for about $40 \mathrm{~min}$ (Scheme 2).

Newly synthesised compounds $\mathbf{5 a}-\mathbf{l}$ were characterised on the basis of elemental analysis, IR, ${ }^{1} \mathrm{H}$ NMR and mass spectral data. The IR spectra of compounds 5a-l showed absorption bands in the range from 3490-2920 $\mathrm{cm}^{-1}$ due to $-\mathrm{CH}_{2}$ - stretching, $\mathrm{C}-\mathrm{H}$ arom. and $\mathrm{NH}$. The strong band 1740 and band at $1690 \mathrm{~cm}^{-1}$ is attributed to the $\mathrm{C}=\mathrm{O}$ stretching vibration. The absorption band seen at a $1600-1560 \mathrm{~cm}^{-1}$ could be attributed to the $\mathrm{C}=\mathrm{N}$ stretching. The weak absorption band showed in the range $681-639 \mathrm{~cm}^{-1}$ is attributed to the (C-S-C) stretching vibration.

The ${ }^{1} \mathrm{H}-\mathrm{NMR}$ spectra of 5a-l showed singlet in the range 5.56-5.67 ppm, which is characteristic for $\mathrm{S}-\mathrm{CH}=\mathrm{C}$ - from 1,3,4-thiadiazine ring. The combined spectral data gave strong support to the proposed structures of all the synthesized compounds.

Scheme 2. Synthetic path for compounds 5a-l.

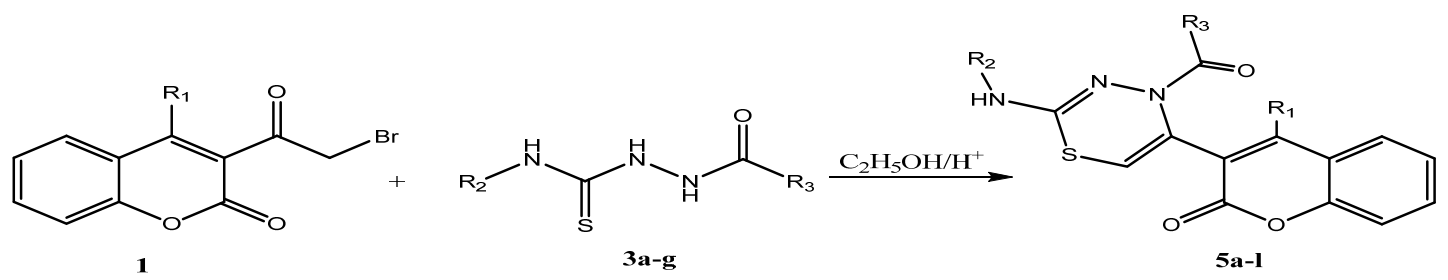

$\mathrm{R}_{1}$ : a) -H; b) -OH 
Scheme 2. Cont.

\begin{tabular}{|c|c|c|c|}
\hline Compound & $\mathbf{R}_{1}$ & $\mathbf{R}_{2}$ & $\mathbf{R}_{\mathbf{3}}$ \\
\hline $5 \mathbf{a}$ & $\mathrm{H}$ & $\mathrm{CH}_{3}$ & \\
\hline $5 b$ & $\mathrm{H}$ & $\mathrm{CH}_{2} \mathrm{CH}_{3}$ & ibid. \\
\hline $5 c$ & $\mathrm{H}$ & $\mathrm{C}_{6} \mathrm{H}_{5}$ & ibid. \\
\hline $5 d$ & $\mathrm{H}$ & $\mathrm{CH}_{3}$ & \\
\hline $\begin{array}{l}5 e \\
5 f\end{array}$ & $\begin{array}{l}\mathrm{H} \\
\mathrm{OH}\end{array}$ & $\begin{array}{l}\mathrm{CH}_{2} \mathrm{CH}_{3} \\
\mathrm{CH}_{3}\end{array}$ & \\
\hline $5 g$ & $\mathrm{OH}$ & $\mathrm{CH}_{2} \mathrm{CH}_{3}$ & ibid. \\
\hline $5 \mathrm{~h}$ & $\mathrm{OH}$ & $\mathrm{C}_{6} \mathrm{H}_{5}$ & ibid. \\
\hline $5 \mathbf{i}$ & $\mathrm{OH}$ & $\mathrm{CH}_{3}$ & \\
\hline $5 \mathbf{j}$ & $\mathrm{OH}$ & $\mathrm{CH}_{2} \mathrm{CH}_{3}$ & ibid \\
\hline $5 k$ & $\mathrm{OH}$ & $\mathrm{C}_{6} \mathrm{H}_{5}$ & $i b$ \\
\hline 51 & $\mathrm{OH}$ & $\mathrm{C}_{6} \mathrm{H}_{5}$ & \\
\hline
\end{tabular}

\subsection{DPPH-Scavenging Activity}

The DPPH free radical exhibits a strong absorption maximum at $517 \mathrm{~nm}$, which is purple in color. Upon the addition of an antioxidant a reduction of DPPH radical to DPPH-H occurs and the color turns from purple to yellow. The method used in this work determines the antioxidant capacity by measuring the remaining amount of DPPH after $30 \mathrm{~min}$ of incubation. This DPPH-scavenging activity was compared to ascorbic acid as a standard compound.

As it can be seen from Table 1, the thiadiazine derivatives are excellent antioxidants. The best ones are compounds $\mathbf{5 b}, \mathbf{5 f}, \mathbf{5 j}$ and $\mathbf{4 b}$, possessing DPPH scavenging activity in a range of ascorbic acid. All of these compounds incorporate a $-\mathrm{OH}$ group in their structure- $\mathbf{5 f}, \mathbf{5 j}$ and $\mathbf{4 b}$ in position 4 of coumarin core and $\mathbf{5 b}$ on the coumarin core linked to the 1,3,4-thaidiazine moiety of the compound. Compounds $\mathbf{5 g}, \mathbf{5 e}, \mathbf{5 h}$ and $\mathbf{5 l}$ also exhibit very high DPPH scavenging activity, in a range $80 \%-90 \%$, but lower than that of the compounds mentioned above. From their structure it is evident that they all, except for compound $\mathbf{5 e}$, bear two hydroxyl groups in their structure. Compounds $\mathbf{5 i}$ and $\mathbf{5 k}$ exhibit DPPH scavenging activity $>70 \%$, and the other compounds did not show significant activity. Since these compounds are complex structures, most of them even containing two coumarin cores, their activity is probably highly dependent on their stereochemistry. 
Table 1. DPPH scavenging activity and iron chelating activity of thiadiazine derivatives ${ }^{a}$.

\begin{tabular}{ccc}
\hline Compound & $\begin{array}{c}\text { \% DPPH scavenging } \\
\text { activity }\end{array}$ & $\begin{array}{c}\text { \% Chelating } \\
\text { activity }\end{array}$ \\
\hline ascorbic acid $^{\mathrm{b}}$ & $85.2 \pm 6.1$ & - \\
EDTA $^{\mathrm{c}}$ & - & $90.9 \pm 7.22$ \\
4a & $17.2 \pm 0.59$ & 0.0 \\
$4 \mathrm{~b}$ & $90.0 \pm 1.24$ & $15.1 \pm 4.27$ \\
$5 \mathrm{a}$ & $36.0 \pm 2.07$ & - \\
$5 \mathrm{~b}$ & $94.4 \pm 1.73$ & 0 \\
$5 \mathrm{c}$ & $17.7 \pm 1.26$ & 0,0 \\
$5 \mathrm{~d}$ & $33.3 \pm 0.27$ & - \\
$5 \mathrm{e}$ & $80.7 \pm 1.61$ & 0.0 \\
$5 \mathrm{f}$ & $94.1 \pm 1.35$ & 0.0 \\
$5 \mathrm{~g}$ & $82.3 \pm 0.70$ & 0.0 \\
$5 \mathrm{~h}$ & $80.6 \pm 0.98$ & $62.5 \pm 2.25$ \\
$5 \mathrm{i}$ & $79.7 \pm 1.29$ & $5.3 \pm 0.56$ \\
$5 \mathrm{j}$ & $90.0 \pm 1.08$ & 53.5 \\
$5 \mathrm{k}$ & $77.2 \pm 0.93$ & $5.18 \pm 0.81$ \\
$5 \mathrm{l}$ & $80.2 \pm 0.44$ & $10.2 \pm 0.42$ \\
\hline
\end{tabular}

${ }^{a}$ data are means \pm standard deviation of three replicates; ${ }^{b}$ ascorbic acid was used as standard in DPPH scavenging activity determination; ${ }^{\mathrm{c}}$ EDTA was used as standard in iron chelating activity determination.

\subsection{Iron Chelating Activity}

This assay is based upon the complex formation between ferrozine and $\mathrm{Fe}^{2+}$, which is red in color. In the presence of chelating agents, the complex formation is disrupted and the red color of the complex is decreased, which can be monitored by spectrophotometric measurement of the color reduction at $562 \mathrm{~nm}$.

Iron is known to be one of the most important lipid oxidation prooxidants due to its high reactivity and may participate in hydroxyl radical generating Fenton type reactions. Thus effective ferrous ion chelators may be important in protection against oxidative damage by removing ferrous ion $\left(\mathrm{Fe}^{2+}\right)[22]$. The most potent iron chelators amongst thiadiazine derivatives investigated in our research are shown to be compounds $\mathbf{5} \mathbf{h}$ and $\mathbf{5} \mathbf{j}$, while other compounds did not show significant activity.

\subsection{Reducing Power}

This assay is based on the reduction of $\mathrm{Fe}^{3+}$ from potassium ferricyanide to $\mathrm{Fe}^{2+}$ (potassium ferrocyanide), which then reacts with ferric chloride to form a ferric/ferrous complex with an absorption maximum at $700 \mathrm{~nm}[23,24]$. A higher absorbance indicates better reducing power of the compound. Compounds possessing the highest reducing power in this assay were $\mathbf{4 b}, \mathbf{5 g}, \mathbf{5 d}$, but their activity was not comparable to that of ascorbic acid, which was used as standard compound (Figure 1). 
Figure 1. Reducing power of tested thiadiazine derivatives.

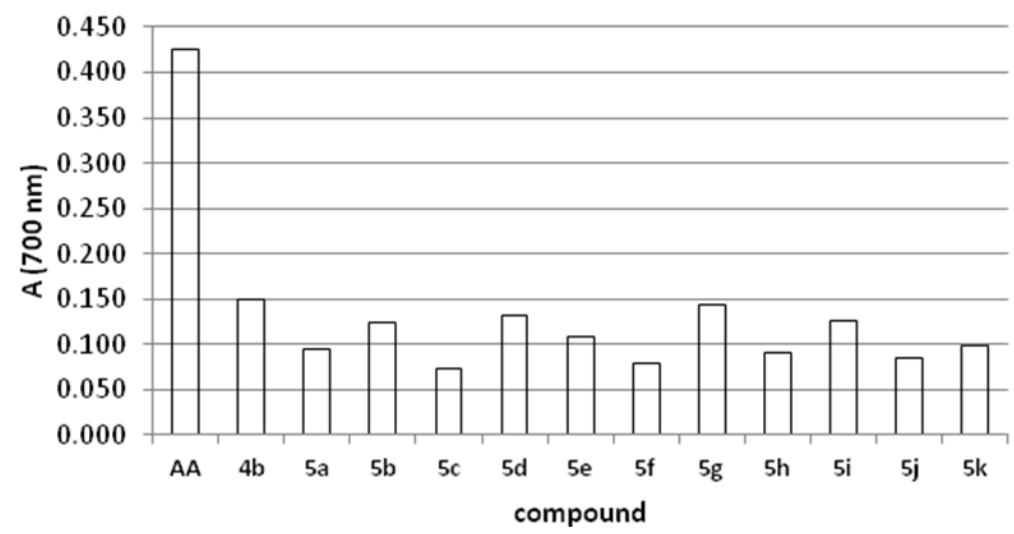

\subsection{Antifungal Activity}

The antifungal activity of thiadiazine derivatives strongly depends on the fungal species. The best antifungal agent on A. flavus (Table 2) was proven to be compound 5e, while observing the overall antifungal activity results, compared to other molds A. flavus was the most resistant to the tested compounds.

Table 2. Minimal inhibitory concentration for $50 \%$ cell death for the tested thiadiazine derivatives.

\begin{tabular}{ccccc}
\hline \multicolumn{5}{c}{ MIC $_{\mathbf{5 0}}$} \\
\hline Compound & A. flavus & A. ochraceus & F. graminearum & F. verticillioides \\
\hline $\mathbf{4 a}$ & $/$ & 0.01 & 0.01 & 0.1 \\
$\mathbf{5 a}$ & 1 & 0.1 & 0.1 & 0.1 \\
$\mathbf{5 b}$ & $/$ & 0.1 & 0.01 & 0.1 \\
$\mathbf{5 c}$ & 0.1 & 0.01 & 0.01 & 0.1 \\
$\mathbf{5 d}$ & 0.1 & 0.1 & 0.01 & 1 \\
$\mathbf{5 e}$ & 0.01 & 0.1 & 0.01 & 0.1 \\
$\mathbf{5 f}$ & 0.1 & 0.1 & 0.01 & 0.1 \\
$\mathbf{5 g}$ & 1 & 0.1 & 0.01 & 0.01 \\
$\mathbf{5 i}$ & 1 & 0.1 & 0.01 & 1 \\
$\mathbf{5 j}$ & 1 & 0.1 & 0.01 & 0.1 \\
\hline
\end{tabular}

Compounds $\mathbf{4 a}$ and $\mathbf{5 c}$ were the best antifungals on A. ochraceus, and compound $\mathbf{5 g}$ showed the best antifungal activity on $F$. verticillioides. $F$. graminearum was the most susceptible mold to the tested compounds and most of the compounds showed high antifungal activity towards this mold. This kind of behavior was also observed in our previous research [15], which could be important since this mold was found to be a potential threat in production of malt barley and wheat in our region [25].

\section{Experimental}

\subsection{General}

Melting points were determined on a capillary melting point apparatus (Electrotermal, Rochford, Great Britain) and are uncorrected. Thin-layer chromatography was performed with fluorescent silica 
gel plates $\mathrm{HF}_{254}$ (Merck, Darmstadt, Germany), which were checked under UV (254 and $365 \mathrm{~nm}$ ) light, using benzene:acetone:acetic acid $(8: 1: 1)$ as a solvent. The elemental analysis for $\mathrm{C}, \mathrm{H}$ and $\mathrm{N}$ was done on a Analyzer 2440 (Perkin-Elmer, Boston, MA, USA). Infrared spectra $\left(v_{\max } / \mathrm{cm}^{-1}\right)$ were recorded on a FT-IR 3303 spectrometer (Beckman Instruments, Inc., Irvine, CA, USA), using KBr disks. ${ }^{1} \mathrm{H}-\mathrm{NMR}$ spectra were recorded on a Bruker Avance $600 \mathrm{MHz}$ NMR Spectrometer (Bruker Biospin GmbH, Rheinstetten, Germany) at $293 \mathrm{~K}$ in DMSO-d6. The MS spectra were recorded on LC/MS/MS API 2000 (Applied Biosystems/MDS SCIEX, Foster City, CA, USA). The absorbance was measured on a UV visible spectrophotometer Heliosy, (Thermo Spectronic, Cambridge, UK). Microplates were read on a Sunrise absorbance reader (Tecan Group Ltd., Männedorf, Switzerland). Incubation was carried in an Aqualytic AL 500-8 incubator (Aqualytic, Dortmund, Germany).

\subsection{Synthesis}

\subsubsection{General Procedure for the Preparation of 1,4-Disubstituted Thiosemicarbazides 3 [21]}

To a solution of corresponding carbohydrazide $(1 \mathrm{mmol})$ in ethanol $(5-10 \mathrm{~mL})$, alkyl/aryl isothiocyanate $(1 \mathrm{mmol})$ and sodium hydroxide $(40 \mathrm{mg}, 1 \mathrm{mmol}$, as a $2 \mathrm{~N}$ solution) were added. The mixture was refluxed for $2-4 \mathrm{~h}$. The precipitate was filtered and crystallized from ethanol/water, to give compounds $3 \mathbf{a}-\mathbf{g}$ in $65 \%-85 \%$ yield.

\subsubsection{Synthesis of 5-(2-Oxo-2H-chromen-3-yl)-6H-1,3,4-thiadizin-2-aminium Bromide (4a)}

A mixture of 3- $\alpha$-bromoacetylcoumarin $(1 \mathrm{a}, 0.01 \mathrm{~mol})$ and thiosemicarbazide $(0.01 \mathrm{~mol})$ was suspended in ethanol $(35 \mathrm{~mL})$ at $0{ }^{\circ} \mathrm{C}$. The mixture was allowed to warm up to room temperature overnight under stirring. The resulting slurry was cooled to $-18{ }^{\circ} \mathrm{C}$ and the precipitate was collected by filtration, washed with cold ethanol, and dried under vacuum. The yellow solid was again suspended in ethanol $(25 \mathrm{~mL})$ which contained $48 \%$ aqueous hydrobromic acid $(1 \mathrm{~mL})$. The reaction mixture was heated to reflux for $45 \mathrm{~min}$. and then allowed to cool down overnight to room temperature. The precipitate was filtered, recrystallized from ethanol, and dried over phosphorus pentoxide under high-vacuum at $40{ }^{\circ} \mathrm{C}$. Yellow crystals $\left(70 \%\right.$ yield); mp $240^{\circ} \mathrm{C}, \mathrm{R}_{\mathrm{f}}=0.52$; FT-IR $v_{\max } 3426,3183,1714,1696,1617,1557$, 1451, 1367, 1250, 1179, 965, 841, 756 and $675 \mathrm{~cm}^{-1}$; ${ }^{1} \mathrm{H}-\mathrm{NMR}$ (DMSO- $\left.d_{6}\right) \delta$ (ppm): 3.91 (s, 2H, $\mathrm{CH}_{2}$ ), 4.80 (s,2H, $\mathrm{NH}_{2} /-\mathrm{N}^{+} \mathrm{H}_{3}$ ), 7.02-7.27 (m, 4H, coum.), 7.45 (s, 1H, H-4 coum); MS m/z 339.0 $[\mathrm{M}-\mathrm{H}]^{+},(\mathrm{M}=340.2)$; Anal. Calcd. for $\mathrm{C}_{12} \mathrm{H}_{10} \mathrm{BrN}_{3} \mathrm{O}_{2} \mathrm{~S}: \mathrm{C}, 42.37$; H, 2.96; N, 12.35\%; Found: C, 42.39; H, 2.94; N, 12.37\%.

\subsubsection{Synthesis of 3-(2-Amino-6H-1,3,4-thiadiazin-5-yl)-4-hydroxy-2H-chromen-2-one (4b)}

Compound 4b was prepared by heating of 3- $\alpha$-bromoacetylcoumarin (1b, 0.01 mol) with thiosemicarbazide $(0.01 \mathrm{~mol})$ in ethanol $(40 \mathrm{~mL})$ with a few drops of hydrochloric acid for $20 \mathrm{~min}$. The reaction mixture was cooled and adjusted to $\mathrm{pH} 8-9$ by adding a dilute solution of ammonia. A brown colored precipitate was formed and filtered off, then recrystallized from $30 \%$ ethanol, and dried under high-vacuum at $40{ }^{\circ} \mathrm{C}$ over phosphorus pentoxide. Pale brown crystals (75\% yield); mp $210{ }^{\circ} \mathrm{C}$; $\mathrm{R}_{\mathrm{f}}=0.66$; FT-IR $v_{\max } 3416,3143,1687,1656,1571,1557,1410,1257,1110,965,891,758$ and $678 \mathrm{~cm}^{-1}$. ${ }^{1} \mathrm{H}-\mathrm{NMR}\left(\mathrm{DMSO}-d_{6}\right) \delta(\mathrm{ppm}): 3.91\left(\mathrm{~s}, 2 \mathrm{H}, \mathrm{CH}_{2}\right), 4.2\left(\mathrm{~s}, 2 \mathrm{H}, \mathrm{NH}_{2}\right), 7.02-7.27$ (m, 4H, coum.), 7.45 (s, 
1H, H-4 coum.), 14.68 (s, $1 \mathrm{H},-\mathrm{OH}) ; \mathrm{MS} \mathrm{m} / z$ 274.01[M-H] $]^{+},(\mathrm{M}=275.04)$; Anal. Calcd. for $\mathrm{C}_{12} \mathrm{H}_{9} \mathrm{~N}_{3} \mathrm{O}_{3} \mathrm{~S}$ : C, 52.36; H, 3.30; N, 15.26\%; Found: C, 52.39; H, 3.29; N, 15.24\%.

3.2.4. General Procedure for Preparation 2,4,5-Trisubstituted 4H-1,3,4- thiadiazines 5a-l

Compounds 5a-l were prepared by heating 3- $\alpha$-bromoacetylcoumarin 1a,b $(0.01$ mol) with the corresponding 1,4-disubstituted thiosemicarbazide $\mathbf{3 a}-\mathbf{g}(0.01 \mathrm{~mol})$ in ethanol $(40 \mathrm{~mL})$ with a few drops of hydrochloric acid for $40 \mathrm{~min}$. The reaction mixture was cooled and made alkaline with a diluted solution of ammonia to adjust the $\mathrm{pH}$ to $8-9$. The precipitate formed was filtered off, recrystallized from $30 \%$ water-ethanol, and dried under high-vacuum at $40{ }^{\circ} \mathrm{C}$ over phosphorus pentoxide.

3-(4-(2-(7-Hydroxy-2-oxo-2H-chromen-4yl)acetyl)-2-(methylamino)-4H-1,3,4-thiadiazin-5-yl)-2Hchromen-2-one (5a). Brown crystals (48\% yield); $\mathrm{mp} 262{ }^{\circ} \mathrm{C} ; \mathrm{R}_{\mathrm{f}}=0.56$; FT-IR $v_{\max } 3227,2979,1710$, $1682,1607,1560,1478,1395,1330,1209,1139,959,843,759$ and $639 \mathrm{~cm}^{-1} ;{ }^{1} \mathrm{H}$ NMR (DMSO- $\left.d_{6}\right) \delta$ (ppm): $2.72\left(\mathrm{~d}, 3 \mathrm{H}, \mathrm{CH}_{3}\right), 3.85$ (s, 2H, $\left.\mathrm{CH}_{2}\right), 4.80$ (q, 1H, NH), 5.56 (s, 1H, thiadiaz.), 6.04-7.37 (m, $9 \mathrm{H}$, coum. $10.86(\mathrm{~s}, 1 \mathrm{H}, \mathrm{OH}), \mathrm{MS} m / z$ 475.1[M-H] $]^{+},(\mathrm{M}=475.5)$; Anal. Calcd. for $\mathrm{C}_{24} \mathrm{H}_{17} \mathrm{~N}_{3} \mathrm{O}_{6} \mathrm{~S}$ : C, 60.63 ; H, 3.60; N, 8.84; Found: C, 60.59; H, 3.60; N, 8.81\%.

3-(2-(Ethylamino)-4-(2-(7-hydroxy-2-oxo-2H-chromen-4yl)acetyl)-4H-1,3,4-thiadiazin-5-yl)-2Hchromen-2-one (5b). Brown crystals (52\% yield); mp $194{ }^{\circ} \mathrm{C} ; \mathrm{R}_{\mathrm{f}}=0.54$; FT-IR $v_{\max } 3492,3134,2978$, $1708,1673,1607,1566,1552,1478,1392,1210,1062,854,759$ and $639 \mathrm{~cm}^{-1}$; ${ }^{1} \mathrm{H}-\mathrm{NMR}$ (DMSO- $d_{6}$ ) $\delta$ (ppm): 2.42 (t, 3H, CH $), 2.69$ (q, 2H, CH 2$), 2.85\left(\mathrm{~s}, 2 \mathrm{H}, \mathrm{CH}_{2}\right), 4.90(\mathrm{t}, 1 \mathrm{H}, \mathrm{NH}), 5.0(\mathrm{~s}, 1 \mathrm{H}, \mathrm{OH}), 5.60$ (s, 1H, thiadiaz.), 6.04-7.37 (m, 9H, coum.), 11.20 (s, 1H, OH); MS m/z 489.1[M-H] $]^{+},(\mathrm{M}=489.5)$; Anal. Calcd. for $\mathrm{C}_{25} \mathrm{H}_{19} \mathrm{~N}_{3} \mathrm{O}_{6} \mathrm{~S}$ : C, 61.34; H, 3.91; N, 8.58; Found: C, 61.32; H, 3.89; N, 8.53\%.

3-(4-(2-(7-Hydroxy-2-oxo-2H-chromen-4yl)acetyl)-2-(phenylamino)-4H-1,3,4-thiadiazin-5-yl)-2H-

chromen-2-one $(\mathbf{5 c})$. Pale grey crystals $\left(62 \%\right.$ yield); mp $252{ }^{\circ} \mathrm{C} ; \mathrm{R}_{\mathrm{f}}=0.51$; FT-IR $v_{\max } 3350,3231$, 3052, 1733, 1691, 1608, 1570, 1457, 1395, 1310, 1136, 1095, 996, 841, 759 and $693 \mathrm{~cm}^{-1}$; ${ }^{1} \mathrm{H}-\mathrm{NMR}$ $\left(\mathrm{DMSO}-d_{6}\right) \delta(\mathrm{ppm}): 2.85\left(\mathrm{~s}, 2 \mathrm{H}, \mathrm{CH}_{2}\right), 4.6(\mathrm{~s}, 1 \mathrm{H}, \mathrm{NH}), 5.67$ (s, 1H, thiadiaz.), 6.04-7.37 (m, 14H, coum. and arom.), $10.52(\mathrm{~s}, 1 \mathrm{H}, \mathrm{OH}) ; \mathrm{MS} \mathrm{m} / z$ 537.1[M-H] $]^{+},(\mathrm{M}=537.5)$; Anal. Calcd. for $\mathrm{C}_{29} \mathrm{H}_{19} \mathrm{~N}_{3} \mathrm{O}_{6} \mathrm{~S}$ : C, 64.80; H, 3.56; N, 7.82; Found: C, 64.79; H, 3.52; N, 7.80\%.

7-(2-(2-(Methylamino)-5-(2-oxo-2H-chromen-2-oxoethoxy)-3-yl)-4H-1,3,4-thiadiazin-4-yl)-4-methyl$2 H$-chromen-2-one (5d). Pale grey crystals $\left(72 \%\right.$ yield); $m p 205{ }^{\circ} \mathrm{C} ; \mathrm{R}_{\mathrm{f}}=0.51$; FT-IR $v_{\max } 3435,3075$, 2970, 2830, 1715, 1677, 1610, 1558, 1449, 1391, 1284, 1213, 1183, 1080, 982, 852, 765 and 684 $\mathrm{cm}^{-1}$; ${ }^{1} \mathrm{H}-\mathrm{NMR}\left(\mathrm{DMSO}-d_{6}\right) \delta(\mathrm{ppm}): 1.71\left(\mathrm{~s}, 3 \mathrm{H}, \mathrm{CH}_{3}\right), 2.74\left(\mathrm{~d}, 3 \mathrm{H}, \mathrm{NCH}_{3}\right), 4.83\left(\mathrm{~s}, 2 \mathrm{H}, \mathrm{OCH}_{2}\right), 5.1$ (q, 1H, NH), 5.64 (s, 1H, thiadiaz.), 5.90-7.28 (m, 8H, coum.); MS $m / z 488.1[\mathrm{M}-\mathrm{H}]^{+},(\mathrm{M}=489.5)$; Anal. Calcd. for $\mathrm{C}_{25} \mathrm{H}_{19} \mathrm{~N}_{3} \mathrm{O}_{6} \mathrm{~S}$ : C, 61.34; H, 3.91; N, 8.58; Found: C, 61.32; H, 3.90; N, 8.53\%.

7-(2-(2-(Ethylamino)-5-(2-oxo-2H-chromen-3-yl)-4H-1,3,4-thiadiazin-4-yl)-2-oxoethoxy)-4-methyl$2 H$-chromen-2-one (5e). Pale grey crystals (73\% yield); $\mathrm{mp} 256{ }^{\circ} \mathrm{C} ; \mathrm{R}_{\mathrm{f}}=0.52$; FT-IR $v_{\max } 3434,3174$, $3029,2734,1765,1715,1607,1556,1449,1391,1277,1153,1072,852,759$ and $681 \mathrm{~cm}^{-1}$; ${ }^{1} \mathrm{H}-\mathrm{NMR}$ $\left(\mathrm{DMSO}-d_{6}\right) \delta$ (ppm): $1.20\left(\mathrm{t}, 3 \mathrm{H}, \mathrm{CH}_{3}\right), 1.74\left(\mathrm{~s}, 3 \mathrm{H}, \mathrm{CH}_{3}\right), 2.69$ (q, 2H, $\left.\mathrm{CH}_{2}\right), 4.83\left(\mathrm{~s}, 2 \mathrm{H}, \mathrm{OCH}_{2}\right)$, 
5.21(q, 1H, NH), 5.60 (s, 1H, thiadiaz.), 5.90-7.28 (m, 9H, coum.); MS m/z 502.1[M-H] $]^{+},(\mathrm{M}=$ 503.5); Anal. Calcd. for $\mathrm{C}_{26} \mathrm{H}_{21} \mathrm{~N}_{3} \mathrm{O}_{6} \mathrm{~S}$ : C, 62.02; H, 4.20; N, 8.35; Found: C, 61.92; H, 4.22; N, 8.30\%.

4-Hydroxy-3-(4-(2-(7-hydroxy-2-oxo-2H-chromen-4-yl)acetyl)-2-(methylamino)-4H-1,3,4-thiadiazin5-yl)-2H-chromen-2-one (5f). Pale yellow crystals $\left(68 \%\right.$ yield); mp $222{ }^{\circ} \mathrm{C} ; \mathrm{R}_{\mathrm{f}}=0.44$; FT-IR $v_{\max }$ 3449, 3337, 1707, 1692, 1613, 1560, 1448, 1395, 1269, 1139, 1032, 853, 759 and $693 \mathrm{~cm}^{-1}$; ${ }^{1} \mathrm{H}-\mathrm{NMR}$ (DMSO-d $d_{6} \delta$ (ppm): $2.70\left(\mathrm{~d}, 3 \mathrm{H}, \mathrm{CH}_{3}\right), 2.85\left(\mathrm{~s}, 2 \mathrm{H}, \mathrm{CH}_{2}\right), 4.89$ (q, 1H, NH), 5.65 (s, 1H, thiadi.), 6.04-7.28 (m, 8H, coum.), 10.80 (s, 1H, OH-7 coum), 15.0 (s, 1H, OH-4 coum); MS m/z 490.1 $[\mathrm{M}-\mathrm{H}]^{+},(\mathrm{M}=491.5)$; Anal. Calcd. for $\mathrm{C}_{24} \mathrm{H}_{17} \mathrm{~N}_{3} \mathrm{O}_{7} \mathrm{~S}: \mathrm{C}, 58.65 ; \mathrm{H}, 3.49 ; \mathrm{N}, 8.55$; Found: C, 58.62; $\mathrm{H}$, $3.52 ; \mathrm{N}, 8.53 \%$.

4-Hydroxy-3-(4-(2-(7-hydroxy-2-oxo-2H-chromen-4-yl)acetyl)-2-(ethylamino)-4H-1,3,4-thiadiazin-5yl)-2H-chromen-2-one (5g). Pale yellow crystals (72\% yield); $\mathrm{mp} 261{ }^{\circ} \mathrm{C} ; \mathrm{R}_{\mathrm{f}}=0.53$; FT-IR $v_{\max } 3447$, 3413, 2986, 1708, 1688, 1615, 1562, 1550, 1392, 1269, 1139, 1032, 829, 758 and $690 \mathrm{~cm}^{-1}$; ${ }^{1} \mathrm{H}-\mathrm{NMR}$ $\left(\mathrm{DMSO}-d_{6}\right) \delta(\mathrm{ppm}): 1.30\left(\mathrm{t}, 3 \mathrm{H}, \mathrm{CH}_{3}\right), 2.69\left(\mathrm{q}, 2 \mathrm{H}, \mathrm{CH}_{2}\right), 2.85\left(\mathrm{~s}, 2 \mathrm{H}, \mathrm{CH}_{2}\right), 5.21(\mathrm{t}, 1 \mathrm{H}, \mathrm{NH}), 5.63(\mathrm{~s}$, 1H, thiadiaz.), 6-04-7.27 (m, 8H, coum.), 10.86 (s, 1H, OH-7 coum), 15.1 (s,1H, OH-4 coum); MS m/z 504.1[M-H] $]^{+},(\mathrm{M}=505.5)$; Anal. Calcd. for $\mathrm{C}_{25} \mathrm{H}_{19} \mathrm{~N}_{3} \mathrm{O}_{7} \mathrm{~S}: \mathrm{C}, 59.40$; H, 3.79; N, 8.31; Found: C, $59.42 ; \mathrm{H}, 3.82 ; \mathrm{N}, 8.34 \%$.

4-Hydroxy-3-(4-(2-(7-hydroxy-2-oxo-2H-chromen-4-yl)acetyl)-2-(phenylamino)-4H-1,3,4-thiadiazin5-yl)-2H-chromen-2-one (5h). Pale yellow crystals $\left(85 \%\right.$ yield); mp $289{ }^{\circ} \mathrm{C} ; \mathrm{R}_{\mathrm{f}}=0.65$; FT-IR $v_{\max }$ 3418, 3184, 1703, 1608, 1548, 1511, 1393, 1322, 1269, 1139, 1034, 852, 758 and $678 \mathrm{~cm}^{-1}$; ${ }^{1} \mathrm{H}-\mathrm{NMR}$ $\left(\mathrm{DMSO}-d_{6}\right) \delta(\mathrm{ppm}): 2.85\left(\mathrm{~s}, 2 \mathrm{H}, \mathrm{CH}_{2}\right), 5.62$ (s, 1H, thiadiaz.), 6.04-7.27 (m, 13H, coum. and arom.), $8.11(\mathrm{~s}, 1 \mathrm{H}, \mathrm{NH}), 15.0$ (s, $1 \mathrm{H}, \mathrm{OH})$; MS $m / z$ 552.1[M-H] $]^{+},(\mathrm{M}=553.5)$; Anal. Calcd. for $\mathrm{C}_{29} \mathrm{H}_{19} \mathrm{~N}_{3} \mathrm{O}_{7} \mathrm{~S}$ : C, 62.92; H, 3.46; N, 7.59; Found: C, 62.89; H, 3.42; N, 7.53\%.

4-Hydroxy-3-(4-(2-(4-methyl-2-oxo-2H-chromen-7-yloxy)acetyl)-2-(methylamino)-4H-1,3,4-thiadiazin-5-yl)-2H-chromen-2-one (5i). Pale yellow crystals $\left(68 \%\right.$ yield); mp $222-223{ }^{\circ} \mathrm{C} ; \mathrm{R}_{\mathrm{f}}=0.67$; FT-IR $v_{\max } 3496,2674,1715,1696,1614,1554,1540,1394,1266,1211,1148,867,759$ and 682 $\mathrm{cm}^{-1} ;{ }^{1} \mathrm{H}$ NMR (DMSO-d $)_{6} \delta(\mathrm{ppm}): 1.71\left(\mathrm{~s}, 3 \mathrm{H}, \mathrm{CH}_{3}\right), 2.73\left(\mathrm{~d}, 2 \mathrm{H}, \mathrm{CH}_{3}-\mathrm{N}\right), 4.83\left(\mathrm{~s}, 2 \mathrm{H}, \mathrm{OCH}_{2}\right), 5.20$ (q, 1H, NH), 5.66 (s, 1H, thiadi.), 5.90-7.28 (m, 8H, coum.), 10.86 (s, 1H, OH-7 coum), 14.6 (s, 1H, OH-4 coum), MS $m / z$ 505.1[M-H] $]^{+},(\mathrm{M}=505.5)$; Anal. Calcd. for $\mathrm{C}_{25} \mathrm{H}_{19} \mathrm{~N}_{3} \mathrm{O}_{7} \mathrm{~S}: \mathrm{C}, 59.40 ; \mathrm{H}, 3.79 ; \mathrm{N}$, 8.31; Found: C, 59.32; H, 3.81; N, 8.30\%.

4-Hydroxy-3-(4-(2-(4-methyl-2-oxo-2H-chromen-7-yloxy)acetyl)-2-(ethylamino)-4H-1,3,4-thiadiazin5 -yl)-2H-chromen-2-one (5j). Pale yellow crystals $\left(78 \%\right.$ yield); mp $226{ }^{\circ} \mathrm{C} ; \mathrm{R}_{\mathrm{f}}=0.69$; FT-IR $v_{\max }$ $3433,3169,2707,1723,1676,1611,1553,1492,1391,1267,1210,1143,1070,988,841,758$ and $681 \mathrm{~cm}^{-1} ;{ }^{1} \mathrm{H}-\mathrm{NMR}\left(\mathrm{DMSO}-d_{6}\right) \delta(\mathrm{ppm}): 1.24\left(\mathrm{~s}, 3 \mathrm{H}, \mathrm{CH}_{3}\right), 1.74\left(\mathrm{t}, 3 \mathrm{H}, \mathrm{CH}_{3}\right), 2.0(\mathrm{~s}, 1 \mathrm{H}, \mathrm{NH}), 2.69$ (q, 2H, $\left.\mathrm{CH}_{2}\right), 4.83$ (s, 2H, $\mathrm{OCH}_{2}$ ), 5.31 (s, 1H, NH), 5.67 (s, 1H, thiadi.), 5.90-7.28 (m, 8H, coum.), 15.0 (s, $1 \mathrm{H}, \mathrm{OH}-4$ coum); MS $m / z$ 518.1[M-H] $]^{+},\left(\mathrm{M}=519.5\right.$ ); Anal. Calcd. for $\mathrm{C}_{26} \mathrm{H}_{21} \mathrm{~N}_{3} \mathrm{O}_{7} \mathrm{~S}: \mathrm{C}, 60.11$; H, 4.07; N, 8.09; Found: C, 60.12; H, 4.02; N, 8.10\%. 
4-Hydroxy-3-(4-(2-(4-methyl-2-oxo-2H-chromen-7-yloxy)acetyl)-2-(phenylamino)-4H-1,3,4-thiadiazin-5-yl)-2H-chromen-2-one (5k). Pale yellow crystals (52\% yield); mp $237-239{ }^{\circ} \mathrm{C} ; \mathrm{R}_{\mathrm{f}}=0.45$; FT-IR $v_{\max } 3466,3424,3064,2920,1707,1651,1612,1543,1496,1448,1387,1266,1151,1075,842$, 755 and $697 \mathrm{~cm}^{-1} ;{ }^{1} \mathrm{H}-\mathrm{NMR}\left(\mathrm{DMSO}-d_{6}\right) \delta(\mathrm{ppm}): 1.71\left(\mathrm{~s}, 3 \mathrm{H}, \mathrm{CH}_{3}\right), 4.83\left(\mathrm{~s}, 2 \mathrm{H}, \mathrm{OCH}_{2}\right), 5.0(\mathrm{q}, 1 \mathrm{H}$, $\mathrm{NH}$ ), 5.60 (s, 1H, thiadiaz.), 5.90-7.27 (m, 13H, coum. and arom.), 15.1 (s, 1H, OH); MS m/z 566.1[M-H] $]^{+},(\mathrm{M}=567.6)$; Anal. Calcd. for $\mathrm{C}_{30} \mathrm{H}_{21} \mathrm{~N}_{3} \mathrm{O}_{7} \mathrm{~S}: \mathrm{C}, 63.49$; H, 3.73; N, 7.40; Found: C, $63.52 ; \mathrm{H}, 3.62 ; \mathrm{N}, 7.32 \%$.

\section{4-Hydroxy-3-(4-(4-hydroxybenzoyl)-2-(phenylamino)-4H-1,3,4-thiadiazin-5-yl)-2H-chromen-2-one}

(51). Ppale yellow crystals (72\% yield); $m p 285{ }^{\circ} \mathrm{C}$; $\mathrm{R}_{\mathrm{f}}=0.69$; FT-IR $v_{\max } 3445,3224,1753,1693$, 1612, 1560, 1532, 1438, 1258, 1103, 963, 845, 756 and $668 \mathrm{~cm}^{-1} ;{ }^{1} \mathrm{H}-\mathrm{NMR}$ (DMSO-d ${ }_{6}$ ) $\delta$ (ppm): 5.20 (s, 1H, NH), 5.66 (s, 1H, thiadi.), 6.46-7.78 (m, 13H, coum. and arom.), $8.90(\mathrm{~s}, 1 \mathrm{H}, \mathrm{OH}), 15.0(\mathrm{~s}, 1 \mathrm{H}$, OH-4 coum.); MS $m / z$ 469.81[M-H] $]^{+},(\mathrm{M}=471.5)$; Anal. Calcd. for $\mathrm{C}_{25} \mathrm{H}_{17} \mathrm{~N}_{3} \mathrm{O}_{5} \mathrm{~S}$ : C, 63.69; H, 3.63; N, 8.91; Found: C, 63.62; H, 3.42; N, 8.70\%.

\subsection{DPPH-Scavenging Activity}

Determination of antioxidant activity was performed according to the procedure described in the literature [26,27] with some modifications described in our previous work [15]. Dimethyl sulfoxide (DMSO) was used as a solvent [28], due to the low solubility of synthesized compounds in ethanol and methanol. A DMSO solution $(750 \mu \mathrm{L})$ of the corresponding thiadiazine derivative $(0.2 \mathrm{mM})$ was added to a DMSO solution of DPPH radical $(0.2 \mathrm{mM})$, so that the final concentration of DPPH radical and the synthesized compound in a solution was $0.1 \mathrm{mM}$. The mixture was shaken and allowed to stand at room temperature. After $30 \mathrm{~min}$ the absorbance at $517 \mathrm{~nm}$ was determined and the scavenging activity was calculated according to the formula below:

$$
\% D P P H \text { scavenging }=\frac{A_{b}+A_{s}-A_{m}}{A_{b}} \times 100
$$

where $\mathrm{A}_{\mathrm{b}}$-absorbance of $0.1 \mathrm{mM}$ DMSO solution of DPPH radical at $517 \mathrm{~nm} ; \mathrm{A}_{\mathrm{s}}$ - absorbance of $0.1 \mathrm{mM}$ DMSO solution of test compound at $517 \mathrm{~nm} ; \mathrm{A}_{\mathrm{m}}$ - absorbance of DMSO mixture of test compound and DPPH radical at $517 \mathrm{~nm}$. Ascorbic acid (AA) was used as a reference compound.

\subsection{Iron Chelating Activity}

The chelating activity of thiadiazine derivatives for ferrous ions $\mathrm{Fe}^{2+}$ was measured according to the literature method [29] with some modifications. $\mathrm{FeCl}_{2}(2 \mathrm{mM}, 25 \mu \mathrm{L})$ were added to $2 \mathrm{mM}$ methanol/DMSO solution $(1 \mathrm{~mL}, 4: 1)$ of the compound investigated. After $30 \mathrm{~s}$, ferrozine ( $5 \mathrm{mM}, 50 \mu \mathrm{L}$ ) was added. Samples were incubated at room temperature for $10 \mathrm{~min}$ and the absorbance of the complex formed between $\mathrm{Fe}^{2+}$ and ferrozine was measured at $562 \mathrm{~nm}$. Metal chelating efficiency of samples was compared to the chelating activity of EDTA disodium salt. The chelating activity of the extract for $\mathrm{Fe}^{2+}$ was calculated as:

$$
\% \text { chelating rate }=\frac{A_{0}-A_{1}}{A_{0}} \times 100
$$


$\mathrm{A}_{0}$ - absorbance of the control (blank, without samples) at $562 \mathrm{~nm} ; \mathrm{A}_{1}$ - absorbance in the presence of the methanol/DMSO sample solution at $562 \mathrm{~nm}$.

\subsection{Reducing Power}

The reducing power of thiadiazine derivatives was determined according to the method [30]. An aliquot of the $0.2 \mathrm{mM}$ thiadiazine derivatives in DMSO $(250 \mu \mathrm{L})$, were mixed with $0.2 \mathrm{M}$ phosphate buffer $(250 \mu \mathrm{L}, \mathrm{pH} 6.6)$ and potassium ferricyanide $\left(\mathrm{K}_{3} \mathrm{Fe}(\mathrm{CN})_{6}, 250 \mu \mathrm{L}, 1 \% \mathrm{w} / \mathrm{v}\right)$. The mixture was incubated at $50{ }^{\circ} \mathrm{C}$ for $20 \mathrm{~min}$, followed by the addition of trichloroacetic acid (TCA, $250 \mu \mathrm{L}, 10 \%$ $\mathrm{w} / \mathrm{v})$, centrifuged for $10 \mathrm{~min}$ at $2000 \times \mathrm{g}$. A portion of the upper layer of the solution $(300 \mu \mathrm{L}) \mathrm{was}$ mixed with distilled water $(300 \mu \mathrm{L})$ and $\mathrm{FeCl}_{3}(0.5 \mathrm{~mL}, 0.1 \% \mathrm{w} / \mathrm{v})$. The absorbance was measured at $700 \mathrm{~nm}$. The higher absorbance indicates stronger reducing power of compound.

\subsection{Antifungal Activity}

\subsubsection{General}

Broth microdilution assays were performed in accordance with the guidelines detailed in CLSI document M38-A [31].

\subsubsection{Tested Fungi}

The fungi used in this experiment (Aspergillus flavus (NRRL 3251); Aspergillus ochraceus (CBS 589.68), Fusarium graminearum (CBS 110.250) and Fusarium verticillioides (CBS 119.825)) are the major producers of mycotoxins and food contaminants [32].

\subsubsection{Preparation of Inoculum}

The inocula were obtained from cultures that were grown on potato dextrose agar (PDA) slants. Aspergillus strains were grown at $35{ }^{\circ} \mathrm{C}$ for 7 days, and Fusarium strains were grown at $35{ }^{\circ} \mathrm{C}$ for 2 days, followed by 7 days at $25{ }^{\circ} \mathrm{C}$. Fungal spores were harvested by pouring $5 \mathrm{ml}$ of sterile saline onto the culture slants and scraping the surface with an inoculation loop. The number of spores in the stock suspension was adjusted to $10^{6}$ spores $/ \mathrm{mL}$, using a Bürker-Türk counting chamber (Haemocytometer). Turbidity of each stock suspension was checked with a 0.5 McFarland standard read at $530 \mathrm{~nm}$. Working $2 \times$ spore suspension was prepared by diluting $200 \mu \mathrm{L}$ of stock suspension into $10 \mathrm{~mL}$ of RPMI 1640 medium [31].

\subsubsection{Medium}

Antifungal susceptibility testing was performed, using RPMI 1640 medium buffered with $0.164 \mathrm{M}$ MOPS (34.53 g/L) and adjusted to $\mathrm{pH} 7.0$ with $(1 \mathrm{M}) \mathrm{NaOH}$, as recommended by the National Committee for Clinical Laboratory Standards. Medium was sterilized by filtration using a $0.45 \mu \mathrm{m}$ filter. 


\subsubsection{Drug Dilutions}

The drug dilutions were prepared following the additive two fold drug dilution scheme described in NCCLS M28-A method for water-insoluble compounds [31]. Briefly, stock drug solutions were first diluted by $20 \times$ of the final concentrations in $100 \%$ dimethyl sulfoxide (DMSO), followed by further dilutions to the final drug concentrations, $10,1,0.1$ and $0.01 \mu \mathrm{g} / \mathrm{mL}$. All drug dilutions were sterilized by filtration using a $0.45 \mu \mathrm{m}$ filter. Sterile polypropylene microtitre plates (96 U-shaped wells; Brand, Wertheim, Germany) were used in the microdilution test. Rows 2-11 contained the series of drug dilutions in $100 \mu \mathrm{L}$ volumes and $100 \mu \mathrm{L}$ of the $2 \times$ spore suspension. Row 1 contained $200 \mu \mathrm{L}$ of uninoculated, drug-free medium and served as the sterility control, while row 12 contained $100 \mu \mathrm{L}$ of drug-free medium and $100 \mu \mathrm{L}$ of inoculum and served as growth control (the final volume in each well was $200 \mu \mathrm{L})$.

\subsubsection{Incubation and MIC Determination}

Following inoculation, all plates were incubated at $35^{\circ} \mathrm{C}$ in an atmospheric incubator. After $48 \mathrm{~h}$ of incubation, plates were read on a microplate reader at $450 \mathrm{~nm}$. Minimal inhibitory concentration for $50 \%$ cell death (MIC 50) was defined as the lowest concentration reducing the optical density by $50 \%$ at $450 \mathrm{~nm}$ compared with growth control [33,34].

\section{Conclusions}

In this paper, and as a part of our ongoing research on biologically active heterocyclic compounds, some thiadiazine derivatives were successfully synthesized and characterized by various spectral methods. Compounds were subjected to antioxidant (DPPH scavenging, iron chelating and reducing power) and antifungal investigation on four mycotoxicogenic fungi, A. flavus, A. ochraceus, $F$. graminearum and $F$. verticillioides namely. The new thiadiazine derivatives were proven to possess an excellent antioxidant activity, comparable to ascorbic acid, while not showing reducing power activity at the same time. Also, most of the compounds were proven to possess potent antifungal activity towards all the tested fungi. Compounds showing the best activity have been selected for further investigation employing some in vivo models.

\section{Conflicts of Interest}

The authors declare no conflict of interest.

\section{References}

1. El-Agrody, A.; Abd El-Latif, M.; El-Hady, N.; Fakery A.; Bedair, A. Hetero aromatization with 4-hydroxycoumarin Part II: Synthesis of some new pyrano[2,3-d]pyrimidines, [1,2,4]triazolo[1,5c]pyrimidines and pyrimido[1,6-b][1,2,4]triazine derivatives. Molecules 2001, 6, 519-527.

2. Rositca, D.N.; Vayssilov, G.N.; Rodios, N.; Bojilova, A. Regio- and stereoselective [2+2] photodimerization of 3-substituted 2-alkoxy-2-oxo-2H-1,2-benzoxaphosphorines. Molecules 2002, 7, 420-432. 
3. Flašík, R.; Stankovičová, H.; Gáplovský, A.; Donovalová, J. Synthesis and study of novel coumarin derivatives potentially utilizable as memory media. Molecules 2009, 14, 4838-4848.

4. Kovalenko, S.; Bylov, I.; Sytnik, K.; Chernykh, V.; Bilokin, Y. A new pathway to 3-hetaryl-2oxo-2H-chromenes: On the proposed mechanisms for the reaction of 3-carbamoyl-2iminochromenes with dinucleophiles. Molecules 2000, 5, 1146-1165.

5. El-Saghier, A.; Khodairy, A.; Khodiyar, A. New synthetic approaches to condensed and spiro coumarins: Coumarin-3-thiocarboxamide as building block for the the synthesis of condense and spiro coumarins. Phosphorus Sulfur. 2000, 160, 105-119.

6. Al-Amiery, A.A.; Al-Bayati, R.; Saour, K.; Radi, M. Cytotoxicity, antioxidant and antimicrobial activities of novel 2-quinolone derivatives derived from coumarins. Res. Chem. Intermediat. 2012, 38, 559-569.

7. Azizian, J.; Mohammadi, A.; Bidar, I.; Mirazaei, P. $\mathrm{KAl}\left(\mathrm{SO}_{4}\right)_{2} \cdot 12 \mathrm{H}_{2} \mathrm{O}$ (alum) a reusable catalyst for the synthesis of some 4-substituted coumarins via Pechmann reaction under solvent-free conditions. Montash. Chem. 2008, 139, 805-808.

8. Satyanarayan, V.S.; Sreevani, P.; Sivakumar, A. Synthesis and antimicrobial activity of new Schiff bases containing coumarin moiety and their spectral characterization. Arkivoc 2008, 17, 221-233.

9. Garazd, M.M.; Muzychka, O.V.; Voyk, A.I.; Nagorichna, I.V.; Ogorodniichuk, A.S. Modified coumarins. 27. Synthesis and antioxidant activity of 3-substituted 5,7-dihydroxy-4methylcoumarins. Chem. Nat. Compd. 2007, 43, 19-23.

10. Smitha, G.; Sanjeeva, R. $\mathrm{ZrCl}_{4}$-catalyzed Pechmann reaction: Synthesis of coumarins under solvent-free conditions. Synth. Commun. 2004, 34, 3997-4003.

11. Kotali, A.; Lafazanis, I.; Harris, P. Synthesis of 6,7-diacylcoumarins via the transformation of a hydroxy into a carbonyl group. Synth. Commun. 2008, 38, 3996-4006.

12. Nofal, Z.M.; El-Zahar, M.; Abd El-Karim, S. Novel coumarin derivatives with expected biological activity. Molecules 2000, 5, 99-113.

13. Kennedy, R.O.; Thornes, R.D. Coumarins: Biology, Applications and Mode of Action; John Wiley and Sons: Chichester, UK, 1997.

14. Zabradnik, M. The Production and Application of Fluorescent Brightening Agents; John Wiley and Sons: New York, NY, USA, 1992.

15. Heravi, M.; Sadjadi, S.; Oskooie, H.; Shoar, R.; Bamoharram, F. The synthesis of coumarin-3-carboxylic acids and 3-acetyl-coumarin derivatives using heteropolyacids as heterogeneous and recyclable catalysts. Catal. Commun. 2008, 9, 470-474.

16. Šarkanj, B.; Molnar, M.; Cacic, M.; Gille, L. 4-Methyl-7-hydroxycoumarin antifungal and antioxidant activity enhancement by substitution with thiosemicarbazide and thiazolidinone moieties. Food Chem. 2013, 139, 488-495.

17. Novikova, A.P.; Perova, N.M.; Chupakhin, O.N. Synthesis and properties of functional derivatives of 1,3,4-thiadiazines and condensed systems based on these compounds (review). Khim. Geterot. Soedin. 1991, 11, 1443-1457.

18. Bose, P.K. Thiodiazines. Part 1. Condensation of thiosemicarbazide with $\omega$-bromo-acetophenone. J. Indian. Chem. Soc. 1924, 1, 51-62. 
19. Pfeiffer, W.D. Methods of Organic Chemistry (Houben-Weyl), 4th ed.; Büchel, K.H., Falbe, J., Hagemann, H., Hanack, M., Klamann, D., Kreher, R., Kropf, H., Regitz, M., Schaumann, E., Eds.; Georg Thieme Verlag: Stuttgart, Germany, 1998; pp. 483-529.

20. Klosa, J. Preparation of 4-hydroxycoumarin ketones with the help of phosphoroxychloride. Arch. Pharm. Ber. Dtsch. Pharmaz. Ges. 1955, 288, 56-61.

21. Gurosi, A.; Karali, N. Synthesis, characterization and primary antituberculosis activity evaluation of 4-(3-Coumarinyl)-3-benzyl-4-thiazolin-2-one benzylidenehydrazone, Turk. J. Chem. 2003, 27, $545-551$.

22. Cacic, M.; Trkovnik, M.; Cacic, F.; Has-Schön, E. Synthesis and antibacterial acivity of some derivatives of (7-hydroxy-2-oxo-2H-chromen-4-yl) acetic acid hydrazide. Molecules 2006, 1, 134-147.

23. Saundaneand, A.R.; Walmik, P. Synthesis, antioxidant, antimicrobial, antimycobacterial, and cytotoxic activities of azetidinone and thiazolidinone moieties linked to indole nucleus. J. Chem. 2013, 2013, 543815:1-543815:9.

24. Singhal, M.; Paul, A.; Singh, H.P.; Dubey, S.K.; Gaur, K. Evaluation of reducing power assay of chalcone semicarbazones. J. Chem. Pharm. Res. 2011, 3, 639-645.

25. Jayanthi, P.; Lalitha, P. Reducing power of the solvent extracts of Eichhornia. crassipes (mart.) solms. Int. J. Pharm. Pharm. Sci. 2011, 3, 126-128.

26. Krstanović, V.; Klapec, T.; Velić, N.; Milaković, Z. Contamination of malt barley and wheat by Fusarium graminearum and Fusarium culmorum from the crop years 2001-2003 in eastern Croatia. Microbiol. Res. 2005, 160, 353-359.

27. Manojkumar, P.; Ravi, T.K.; Subbuchettiar, G. Synthesis of coumarin heterocyclic derivatives with antioxidant activity and in vitro cytotoxic activity against tumor cells. Acta Pharm. 2009, 59, 159-170.

28. Wu, C.R.; Huang, M.Y.; Lin, Y.T.; Ju, H.Y.; Ching, H. Antioxidant properties of Cortex fraxini and its simple coumarins. Food Chem. 2007, 104, 1464-1471.

29. Taskova, R.; Mitova, M.; Mikhova, B.; Duddeck, H. Bioactive phenolics from Carthamus. lanatus, L. Z. Naturforsch. C 2003, 58c, 704-707.

30. Zhao, G.-R., Xiang, Z.-J.; Ye, T.-X.; Yuan, Y.-J.; Guo, Z.-X. Antioxidant activities of Salvia miltiorrhiza and Panax notoginseng. Food Chem. 2006, 99, 767-774.

31. Igbinosa, O.O.; Igbinosa, I.H.; Chigor, V.N.; Uzunuigbe, O.E.; Oyedemi, S.O.; Odjadjare, E.E.; Okoh, A.I.; Igbinosa, E.O. Polyphenolic contents and antioxidant potential of stem bark extracts from Jatropha. curcas (Linn). Int. J. Mol. Sci. 2011, 12, 2958-2971.

32. National Committee for Clinical Laboratory Standards. Reference Method For Broth Dilution Antifungal Susceptibility Testing of Filamentous Fungi; In Approved standard, NCCLS document M38-A; CLSI: Wayne, PA, USA, 2002.

33. Hussein, H.S.; Brasel, J.M. Toxicity, metabolism, and impact of mycotoxins on humans and animals. Toxicology 2001, 167, 101-134. 
34. Clement, M.; Tremblay, J.; Lange, M.; Thibodeau, J.; Belhumeur, P. Purification and identification of bovine cheese whey fatty acids exhibiting in vitro antifungal activity. J. Dairy Sci. 2008, 91, 2535-2544.

35. Santos, M.M.M.; Faria, N.; Iley, J.; Coles, S.J.; Hurrsthouse, M.B.; Martins, M.L. Reaction of naphthoquinones with substituted nitromethanes. Facile synthesis and antifungal activity of naphtho[2,3-d]isoxazole-4,9-diones. Bioorg. Med. Chem. Lett. 2010, 20, 193-195.

Sample Availability: Samples of the compounds $\mathbf{5 a}-\mathbf{I}$ are available from the authors.

(C) 2014 by the authors; licensee MDPI, Basel, Switzerland. This article is an open access article distributed under the terms and conditions of the Creative Commons Attribution license (http://creativecommons.org/licenses/by/3.0/). 